\title{
Timing of coronary artery bypass grafting following acute myocardial infarction
}

\author{
Thomas Yau ${ }^{1}$, Zachary Brigden ${ }^{1}$, and Amer Harky ${ }^{2}$ \\ ${ }^{1}$ Queen Mary University of London \\ ${ }^{2}$ Liverpool Heart and Chest Hospital NHS Foundation Trust
}

July 25, 2020

\begin{abstract}
Objective: There is wide variations in practice about the exact timing of coronary artery bypass grafting (CABG) post-acute myocardial infarction (AMI) and admission to hospital. This study aims to review current literature evidence to provide an up-to-date evaluation of the optimal CABG timing and parameters indicative of patient outcomes. Method: Electronic literature search was done to look into articles that discussed acute myocardial presentation and their referral for CABG either as urgent in-patient or as elective cases. The evidence was synthesised based on each reported article and their outcomes. Results: The timing of CABG following AMI have been explored in multiple studies, however there is no clear consensus on when to proceed with $\mathrm{CABG}$ following $\mathrm{AMI}$ and this remains controversial. The mortality rates vary with timing of $\mathrm{CABG}$, but confounding factors such as age, poor pulmonary functions, renal insufficiency, poor left ventricular function and many others can all contribute to the variable reported outcomes. Conclusion: There is need for large, multi-centre study to identify optimal timings for CABG in cases of complex coronary artery diseases or failed PCI in patients with acute MI. Upcoming guidelines should emphasize patient cohorts by taking their risk factors into consideration.
\end{abstract}

\section{Abstract}

Objective: There is wide variations in practice about the exact timing of coronary artery bypass grafting (CABG) post-acute myocardial infarction (AMI) and admission to hospital. This study aims to review current literature evidence to provide an up-to-date evaluation of the optimal CABG timing and parameters indicative of patient outcomes.

Method: Electronic literature search was done to look into articles that discussed acute myocardial presentation and their referral for CABG either as urgent in-patient or as elective cases. The evidence was synthesised based on each reported article and their outcomes.

Results: The timing of CABG following AMI have been explored in multiple studies, however there is no clear consensus on when to proceed with CABG following AMI and this remains controversial. The mortality rates vary with timing of CABG, but confounding factors such as age, poor pulmonary functions, renal insufficiency, poor left ventricular function and many others can all contribute to the variable reported outcomes.

Conclusion: There is need for large, multi-centre study to identify optimal timings for CABG in cases of complex coronary artery diseases or failed PCI in patients with acute MI. Upcoming guidelines should emphasize patient cohorts by taking their risk factors into consideration.

\section{Introduction}

Cardiovascular disease (CVD) accounts for an estimated $31 \%$ of all annual worldwide deaths. ${ }^{[1]}$ Overtime, the development of atherosclerotic plaques will stenose the coronary vessel wall leading to ischaemia and 
increasing its susceptibility to rupture. Consequently, through thrombotic vessel occlusion, acute coronary syndrome (ACS) will manifest. ACS is one of the end products of CVD and stands as one of the leading causes of death in developed countries. ACS encompasses angina pectoris and acute myocardial infarctions (AMI). ${ }^{[2]}$ AMI can be divided into either ST-segment elevation MI (STEMI) or non-ST-segment elevation MI (nSTEMI). The former is often associated with complete obstruction of coronary vessel from intraluminal thrombosis due to either erosion or sudden rupture of an atheromatous plaque within the coronary walls. ${ }^{[3]}$ While an nSTEMI is the partial occlusion of a coronary vessel from either an enlarged or ruptured atheromatous plaque, leading to ischaemia. Continued ischaemia can lead to cardiac cell death, loss of heart muscle contractility and life-threatening arrhythmias. ${ }^{[2]}$ Revascularisation procedures such as percutaneous coronary intervention (PCI) and coronary artery bypass grafting $(\mathrm{CABG})$ are the gold standard treatment for ACS. However, successful outcome of treatment depends on effective patient selection and appropriate timing of intervention. Landmark randomised control trials (RCTs) such as; SYNTAX, EXCEL and NOBLE studies have identified sub-group of patients that would benefit from one intervention to the other. Although, there is a time delay in the effect associated with CABG, it is able to replace an occluded vessel entirely and treat multiple diffused lesions, while demonstrating greater durability by decreasing revascularisation rates. Usually patients who benefit from CABG than PCI are those with multiple complex comorbidities such as diabetes and multivessel disease. However, practical and financial constraints continue to pose a barrier for CABG management. ${ }^{[4]}$ Often patients have to wait in hospital after an MI for several weeks before they are scheduled for CABG because of backlogging and the long queues for operation theatres [5].

Due to an ageing population, there has been a significant increase in patients with multivessel diseases and associated comorbidities, thus indicating a need for CABG in clinical practice. ${ }^{[6]}$ However, evidence regarding optimal timing as well as factors determining outcomes, need to be addressed to optimise CABG. There is a clear correlation with patient selection and patient outcome, thus evidence pinpointing the target population that would benefit from CABG is welcomed. ${ }^{[7]}$ Additionally, the lack of standardisation in reporting trial outcomes of cardiac surgery has been raised and adds to the confusing nature of the debate. ${ }^{[8]}$ Development of a standardised outcome reporting system would strengthen the process of evidence-based care in cardiac surgery. The purpose of this literature review is to explore the evidence surrounding the timing of CABG reperfusion in STEMI and nSTEMI patients and draw conclusions about the optimal timing to improve outcomes in these patients.

\section{Method}

The literature review was conducted using the electronic database PubMed to find records published between 2005-2020 describing CABG timing and outcomes of post AMI. Records were limited to English and screened for relevant titles/abstracts that reported findings about CABG timing and outcome. Additional findings regarding parameters indicative of CABG patient outcome were also reviewed. Search terms included; CABG, STEMI/nSTEMI, optimal timing and outcome. The evidence was reviewed and synthesised based on each reported article.

\section{PCI and trend of non-elective CABG}

For many years, PCI has been preferred in treating STEMI, due to the relative ease of carrying out this procedure, as well as a quicker treatment time when compared to CABG. ${ }^{[9]}$ During STEMI, the urgent need for reperfusion to reduce the risk of cardiac ischaemia and infarction is critical for patient survival. As shown in table 1, revascularisation strategies such as PCI and CABG are often compared, however in different contexts, different strategies will be indicated. The SYNTAX [Synergy Between PCI With Taxus and $\mathrm{CABG}$ ] score is normally used to stratify CVD patients for either PCI or CABG and ranges from scores 0 to $>60$. According to literature, patients with low SYNTAX score $(<22)$ is of class IIa and a low-intermediate SYNTAX score $(<33)$ is of class IIb, both are indicated for PCI treatment. While patients with a high SYNTAX score ([?]33) exhibit more complex disease and may involve multiple vessels (class I) and these patients will benefit from CABG. ${ }^{[10]}$

A number of studies have raised the importance of timing in the outcome of PCI and CABG for AMI. Various 
outcomes reported are subjected to different timing of interventions. However, regardless of timing, CABG has shown to exhibit greater future benefits such as long-lasting preventive effects, as well as significantly improving health-related quality of life (QoL). ${ }^{[11]}$ While, PCI-treated patients have a lower stroke risk, PCI incur a higher revascularisation rate when compared to CABG-treated patients. CABG surgery remains the gold standard treatment option for nSTEMI patients with significant left main disease (LMD) or triple vessel disease (TVD). ${ }^{[12]}$ CABG significantly reduces the risk of death associated with MI or stroke when compared to PCI with drug-eluting stents. ${ }^{[13]}$ However, CABG is associated with an increased likelihood of cerebrovascular events. ${ }^{[14]}$ Presumably higher incidence of stroke among patients is explained to be from an increased systemic inflammatory state caused by AMI. ${ }^{[15]}$ Thus, PCI is preferred in early revascularisation of ischaemia, rather than opting for non-elective CABG.

Although non-elective CABG is rare and not recommended, in situations where patients who fail to have PCI or PCI is not indicated, urgent CABG will be required as last-line treatment. An emergency CABG for STEMI patients must be performed before a 6 hours lapse after the acute event, otherwise it must be postponed from 1 day to 1 month. ${ }^{[16]}$ Patients undergoing non-elective CABG is associated with longer inhospital stays and increase in healthcare costs, this is mainly due to differences in rates of preoperative stays and additional procedures like angiography and PCI. Moreover, non-elective CABG is reported to increase patients' risk of myocardial infarction when compared to elective CABG. ${ }^{[17]}$

CABG offers survival advantage compared to medical therapy for life-threatening situations such as unstable angina and left ventricular (LV) dysfunction. A recent study reported that CABG achieved lower major adverse cardiac evets (MACE) and decreased mortality in patients with severely reduced LV ejection fraction (LVEF) when compared PCI. ${ }^{[18]}$ As oppose to PCI only treating proximal singular lesions, CABG bypasses the proximal $2 / 3$ of vessel, where current lesion and future threatening lesions may occur. This incurs a protective prognostic effect, whereby exhibiting improved long-term survival in the first 8-years for patients with TVD. ${ }^{[19]}$

\section{Concept of hot CABG}

It is widely accepted that ongoing ischemia, unstable refractory angina, and the burden of CVD may influence the timing of CABG and associated with greater hospital mortality rate (HM). ${ }^{[20]}$ Despite CABG exhibit marked advantages of long-term benefits, disadvantages of major surgical risk and recovery time sways patients to less invasive interventions (PCI or thrombolytic therapy).

In the presence of ischaemia, CABG is indicated for patients within 24 hours of presentation, while postponement of CABG can be up to 4-30 days providing there are anatomical indications. ${ }^{[21]}$ However, CABG is often preferred in the later settings, due to evidence showing an increased in mortality for patients being operated within the first 24 hours to 3 days after diagnosis of STEMI. This preference is supported by evidence showing that operative mortality declines with the delaying of CABG.

Advances in perioperative care and myocardial protection have allowed for postponement of CABG treatment in AMI. ${ }^{[14]}$ Nichols et al. reported that patients undergoing CABG within 1 day of MI had higher rates of STEMI and significantly higher HM. Furthermore, patients who had CABG within 1-2 days and 3-7 days after MI, achieved similar mortality rates, suggesting that delaying CABG is possible without sacrificing outcomes. ${ }^{[20]}$ STEMI patients operated within 3 days, were treated less frequently with PCI and glycoprotein IIb/IIIa inhibitors but displayed greater operative risk as expressed in the European System for Cardiac Operative Risk Evaluation (EuroSCORE). Consequently, patients endured longer intensive care and overall hospital stay. ${ }^{[21]}$ Therefore, it can be inferred that emergency CABG within 3 days for patients with acute STEMI is contraindicated.

Contrastingly, a retrospective study investigating the outcome of haemodynamically stable patients treated with early CABG revealed that, STEMI patients had reduced perioperative complications and better survival compared to nSTEMI patients. Interestingly when CABG was performed within 6 hours, mortality was lower in STEMI patients than nSTEMI patients $(1.8 \%$ vs $\% .1 \%, P=0.041)$. Likewise, a 30-day mortality was significantly lower in STEMI than nSTEMI patients (2.7\% vs 6.6\% P=0.018). Overall, CABG significantly 
improved survival of both STEMI and nSTEMI patients after 1 year ( $94 \%$ vs $88 \%, P<0.001$ ), after 5 years $(87 \%$ vs $73 \%, P<0.001)$ and after 10 years $(74 \%$ vs $57 \%, P<0.001) .{ }^{[22]}$ Hence highlighting the long-term benefits of CABG in treating both STEMI and nSTEMI.

Similarly, early revascularization in STEMI patients without cardiogenic shock was associated with superior short/long-term outcomes than nSTEMI patients. These benefits were identified in significantly younger STEMI patients whom were fast tracked for CABG. This suggests that immediate CABG should be implemented during or soon after coronary angiography. ${ }^{[22]}$

Literature about optimal timing of CABG for treating nSTEMI patients have been conflicting as well. It has been reported that nSTEMI patients who had CABG $<24$ hours achieved significantly better 5-year survival than patients being treated 24-72 hours $(P=0.02)$. Contrastingly, CABG achieved a better 5-year survival, when nSTEMI patients were treated $>72$ hours to 21 days than within $24-72$ hours. ${ }^{[23]}$ Likewise, Parikh et al. argued that early ( $<48$ hours) CABG is non-inferior to late ( $>48$ hours) CABG, hence delaying CABG would be ideal to increase resources without compromising patient outcome. ${ }^{[24]}$

From the literature it can be inferred that appropriate patient selection for CABG is critical for determining a positive long-term outcome for the overall patient population presenting with AMI. From table 2, confounding factors can negatively affect the outcome of CABG and predicative of HM include; poor LVEF, age $>70$ years, prior CABG/treatment modalities, systemic comorbidities and previous history of stroke. ${ }^{[21,25]}$ Assman et al. reported that early CABG after AMI ( $<10$ days) is accompanied with significantly increased mortality, especially in elderly patients or in patients with a severely impaired LVEF. ${ }^{[26]}$ This reinforces that age and LVEF can predict patient recovery from CABG. ${ }^{[18,27]}$

Clinical trials have identified patient population such as diabetics whom would benefit from CABG for revascularisation therapy rather than PCI. ${ }^{[28-30]}$ From the FREEDOM trial, it has been shown that diabetic patients with multivessels disease treated with CABG achieved better QoL and intermediate health status between 6-24 months, although beyond 24 months there were no consistent significant difference observed between CABG and PCI using drug-eluting stents. ${ }^{[31]}$ Interestingly, the positive outcomes of CABG for type 2 diabetic patients are not seen in type 1 diabetics. However, CABG has proved to be superior over PCI in treating ACS patients with type 1 diabetes. Hence an emphasis on preoperative glycaemic control is integral for CABG outcomes in diabetic patients.

\section{Current guidelines:}

The armamentarium available for the treatment of ACS can be split into either pharmaceutical or surgical interventions. Pharmaceuticals include; beta-blockers, anti-hyperlipidaemia drugs, anti-platelet therapy, ACE-inhibitors and hormones. The goal of these treatments is to correct the imbalance of nutrient supply and demand of the heart by lowering its workload, whilst simultaneously increasing blood supply. Alternatively, surgical revascularisation (PCI or CABG) is offered. Patients suspected of AMI are treated immediately with oxygen, glyceryl trinitrate and aspirin as well as undertaking an electrocardiogram (ECG) to diagnose for STEMI or nSTEMI. ${ }^{[32]}$

\subsection{STEMI guidelines:}

Patients diagnosed with STEMI are immediately assessed for their eligibility for surgical reperfusion with ineligible patients being offered pharmaceutical intervention discussed above. The primary PCI (PPCI) pathway is activated in eligible patients who present within 12 hours of developing symptoms and who are able to have PPCI within 120 minutes of fibrinolysis administration. Otherwise, in patients presenting after 12 hours of symptom onset, the pathway is activated only if there is evidence of continued ischaemia. Patients who are unable to have PPCI within 120 minutes of the fibrinolysis administration, should be offered a repeat ECG at 60-90 minutes with follow on PPCI, if an ST-segment-elevation persists. $\left.{ }^{33}, 34\right]$

PPCI has become the most common treatment for STEMI in the UK, with it being used to treat $~ 95 \%$ of cases. $^{[35]}$ In the remaining patients, CABG is the preferred method of reperfusion. A computed tomography coronary angiogram is used to assess a patient's suitability for reperfusion therapy. This is used to identify 
the causative lesion/(s) and corresponding anatomy. Patients are stratified using the SYNTAX score to assess whether PCI or CABG would be most suitable in this patient.

\section{2 nSTEMI guidelines:}

Patients diagnosed with an nSTEMI are immediately offered pharmaceutical intervention (aspirin, heparin, fondaparinux or antithrombin) and stratified into low, medium or high-risk groups using an established scoring system such as the GRACE score. ${ }^{[32]}$ The GRACE score estimates the admission to 6 month mortality based on age, blood pressure, creatinine levels as well as other criteria. ${ }^{[36]}$ Patients with low risk are managed conservatively with continued monitoring. Intermediate and higher risk patients are offered clopidogrel and assessed for reperfusion surgery and given a SYNTAX score. Similar to the management of STEMI, PPCI is the favoured option for reperfusion in the vast majority of patients. CABG is only preferred when PCI is contraindicated in patients with a high SYNTAX score. ${ }^{[37]}$

\subsection{Issues with current guidelines:}

The current guidelines are based on the most up-to-date meta-analysis and scientific reports and their use has correlated with a consistent drop in ACS mortality rates. ${ }^{[38]}$ However, there are still numerous issues and points of contention surrounding the guidelines, that needs to be raised and addressed to improve patient outcomes. Firstly, despite numerous studies showing the benefit of timely PPCI, evidence shows that some areas of the country are still unable to receive PPCI in a timely manner, this could be due to a number of different circumstances including accessibility of Cath labs. ${ }^{[39]}$ Secondly, despite the focus on PPCI as the primary treatment for AMI, the number of CABG surgeries being performed has been consistently rising. [40] It is thought that improvements in diagnosis and screening tools such as GRACE and SYNTAX, have led to the increased recognition of high-risk patients that are more suitably managed by CABG.

\section{STEMI and timing of CABG:}

During our literature search, it became clear that far less research into optimal CABG timing has been carried out on STEMI patients compared to nSTEMI patients. This, along with the challenges already discussed above make drawing meaningful conclusions challenging. For example, many studies that compared early to late intervention did not distinguish between PCI or CABG. Similarly, studies that compared early and late CABG intervention did not distinguish between STEMI and nSTEMI patients.

The current common practice among cardiac surgeons is to delay CABG after STEMI for optimal clinical outcome. This view has formed in light of various studies that show a beneficial effect when CABG is delayed. One study reported that HM decreased when CABG surgery was delayed. $14.2 \%$ ( $<6$ hours), $13.8 \%$ (6 hours to 1 day), $7.9 \%$ (1-3 days), 3.8\% (4-7 days), $2.9 \%$ (7-14 days) and $2.7 \%$ ( $>15$ days). These results supported the recommendation to delay CABG for 3 days in patients when possible. ${ }^{[41]}$ Similarly, other studies showed that early CABG was associated with a higher mortality than late CABG $(5.6 \%$ vs $3.8 \%, P<0.001)$. Furthermore, it was shown that early CABG independently predicted mortality after controlling for clinical acuity and propensity matching (odds ratio $[O R]=1.43, P=0.003$ ). ${ }^{[42]}$ Conversely, other studies reported that there was no difference in the overall mortality of patients receiving early or late CABG for STEMI. However, the incidences of rethoracotomy in these patients significantly changed with CABG timing. Patients operated within 24 hours, 1-3 days, and 4-30 days had rethoracotomy rates of 31\%, $38 \%$ and $5 \%$ respectively. Thus, concluding that the risks of rethoracotomy increased in STEMI patients being treated with CABG within 3 days of presentation. ${ }^{[21]}$

\section{nSTEMI and timing of CABG:}

Although CABG is not the favoured method for surgical revascularisation in the majority of AMIs [32], it remains an important intervention in high-risk patients including those with multivessel disease. ${ }^{[12]}$ Several studies have explored the effect of timing of CABG in patients with nSTEMI. Many of these have shown no significant difference in the overall outcome between early and late CABG. For example, one study compared the primary outcome of death, MI or stroke at 6 months in patients who had early versus late intervention and showed no significant difference $(P=0.15) \cdot{ }^{[43]}$ Likewise, another study showed no significant difference 
in HM (OR: 1.12, 95\% CI: 0.71-1.78) or the composite outcome of death, MI, congestive heart failure or cardiogenic shock (OR: 0.94, 95\% CI: 0.69-1.28) between early and late CABG. ${ }^{[24]}$ Similarly, a study showed no significant difference in the incidence of HM between early ( $<48$ hours) and late ( $>48$ hours) CABG $(P=0.695) .{ }^{[25]}$ However, it is worth noting from the RIDDLE-NSTEMI study comparing early $(<2$ hours) and late ( $<72$ hours) invasive strategy (CABG or PCI) and showed that nSTEMI patients who were treated early achieved lower mortality risks than those treated late, with a significant reduction in risk of subsequent MIs being reported (4.3\% vs $13 \%$ respectively, $H R=0.32,95 \%$ CI: $0.13-0.74, P=0.008$ ). ${ }^{[44]}$ Conversely, another study found that HM was higher in patients who had CABG within 1 day after MI, however no distinction was made between STEMI and nSTEMI. ${ }^{20]}$

However, numerous studies have reported that significant differences exist in secondary outcomes such as bleeding events, ischaemic complications and refractory ischaemia. In the ACCOAST study, patients receiving CABG in less than 2.98 days, between 2.98 - 6.95 days and over 6.95 days were compared. Major bleeding events occurred in $26 \%, 10.4 \%$ and $4.8 \%$ respectively $(P<0.001)$, showing that early revascularisation carried a significantly increased risk of bleeding. ${ }^{45]}$ However, the data gathered from another study showed that there was a significant reduction in the secondary outcome of death, MI or refractory ischaemia in early CABG (9.5\%) compared to late CABG (12.9\%). ${ }^{[4]}$ The disagreement between these results illuminate the complexity and challenges of establishing the optimal timing for CABG post nSTEMI.

\section{Comparison of CABG timing in STEMI and nSTEMI patients:}

With a lack of RCTs, it is difficult to be certain that the outcomes described above are indeed due to the timing of CABG or other confounding factors. The majority of the retrospective studies showed that patients with early or late CABG are often dissimilar in many factors that could influence the outcome. For example, patients who are recognised as being more high-risk (through GRACE or SYNTAX scoring) tend to be treated via CABG earlier than those who are lower risk. Lower risk patients are typically revascularized with PCI and only have adjuvant CABG, if PCI fails. ${ }^{[5]}$ Therefore, acuity of illness could be influencing the results described above. ${ }^{[43]}$ However as discussed above, some studies did not report any significant difference in outcome despite higher-risk patients being treated earlier. ${ }^{[24]}$ Similarly, in studies that propensity matched patients to remove variable clinical features, no significant difference in primary outcome was observed. ${ }^{[25]}$

This evidence for a range of confounding factors influencing CABG outcome, press the case for a more individualised approach to CABG revascularisation. As many different factors have been found to contribute to the clinical outcome of CABG intervention, assessing all these factors in an individual patient and making an informed choice for that patient based on their own unique risk could optimise CABG intervention. Steps towards this have already been taken in recent years with the introduction of the SYNTAX score. ${ }^{[10]}$ Perhaps a similar system could be implemented to aid clinicians in deciding whether a patient will benefit from early or delayed CABG to treat STEMI or nSTEMI.

\section{Factors predictive of outcomes:}

There are clear differences in the data between STEMI and nSTEMI for optimal timing of CABG. The majority of nSTEMI studies show no significant difference between early and late intervention in overall outcome, but a slight preference for early intervention in secondary outcomes such as bleeding complications or acute ischaemia has been observed. In contrast, STEMI studies have shown increased mortality in early CABG compared to late. Despite these apparent differences, research into both types of MIs have revealed a whole host of confounding factors that influence the outcome of the CABG surgery, independent of the time it was performed. Therefore, these findings suggest that a more complex view of the optimal timing for CABG is needed for both STEMI and nSTEMI patients.

Patient characteristics are heavy predicative factors of CABG as shown in table 2. Body mass index (BMI), LMD, and renal insufficiency have all been identified to be independent predictors of long-term mortality in CABG. ${ }^{[23]}$ Pathological signs of disease complexity, STEMI vs nSTEMI, health comorbidities can also radically determine therapeutic pathways. For instance, diabetic patients with chronic kidney disease (CKD) tend to exhibit more MACE after $\mathrm{CABG}^{[46]}$ 
Table 2 also shows that preoperative assessment of pulmonary function can provide prognostic values of CABG, with many patients developing respiratory insufficiency after CABG due to pre-existing chronic obstructive pulmonary disease (COPD). [47] Preoperative administration of dual antiplatelet therapy has shown to achieve protective effect against in-hospital death and decrease in bleeding risks in non-elective CABG. ${ }^{[23]}$ While certain drugs such as clopidogrel can delay CABG intervention for AMI patients. ${ }^{[24]}$ Preoperative measures of elevated biomarkers (C-reactive protein and Troponin I) have also shown to predict subsequent death, MI and stroke after patient revascularisation. ${ }^{[48,49]}$ These finding suggests that appropriate preoperative measures can provide crucial information for improving CABG outcome regardless of CABG timing.

It is clear that despite similar studies are being conducted to identify the optimal timing of CABG, different outcomes are being reported. This could be due to a lack of standardised definitions of early/late intervention and methods in reporting outcomes. Therefore, better diagnostic modalities for aiding the standardisation of definition would yield more convincing data regarding optimisation of CABG. Rather than focussing on the timing of $\mathrm{CABG}$, it can be hypothesised that multiple confounding factors seem to play a bigger role in determining patients' outcome.

\section{Significance of left ventricular contribution and future directions of CABG:}

Reduced LV function and scarring are often the end product of an AMI [50]. It has been observed that complications post-surgery is associated with increased LV scarring/infarct burden. Accordingly, studies have suggested that presence of scar tissue after CABG is predictive of cardiac functional recovery. ${ }^{[51,52]}$

Cardiac Magnetic resonance (CMR) imaging has become gold standard imaging technique for assessment of cardiac volumes, function and mass. Assessment of size and extent of scarring/fibrosis by Late Gadolinium Enhancement (LGE) has aided the detection and assessment of AMI. ${ }^{[53]}$ In a retrospective study investigating the utility of $1.5 \mathrm{~T}$ CMR in predicting immediate and six-week outcomes after CABG surgery patients with high EuroSCORE $(>16)$ and impaired LVEF $(<40 \%)$. Sheriff et al. observed a significant relationship between lower circumferential relaxation index (a CMR parameter) and the occurrence of immediate postoperative complications. ${ }^{[52]}$ Thus, CMR would be suitable for use prior to CABG and provide additional prognostic information. Additionally, CMR can be used for monitoring assessing patient recovery process. It is reported that 6 months after CABG, cardiac segments with scar and no scar showed statistical improvement in functionality, although only a small percentage (6.3\%) showed improved contractility in the former sub-group. ${ }^{[51]}$ The concept of adding CMR-derived parameters into diagnosis may improve the accuracy of risk-scoring systems for clinicians to make informed decisions regarding CABG utility and hence, future research in this area is warranted.

\section{Conclusion:}

From current literature, conflicting finding from studies can be inferred that specific pathology and complexity of disease will affect the outcome of CABG and determine the optimal timing for CABG. Therefore, it is clear that optimising timing of CABG needs to take into account patient characteristics and distinctive pathological status. Cardiac diagnostic modalities like CMR can provide essential information pre/post CABG. In a time of personalised medicine, a need for greater cardiac screening methods and standardised development of scoring systems can aid the optimisation of CABG and accordingly the optimal time for this intervention.

\section{Reference:}

1. WHO. Cardiovascular diseases (CVDs) . 2017; Available from: https://www.who.int/en/news-room/factsheets/detail/cardiovascular-diseases-(cvds).

2. Mechanic, O.J. and S.A. Grossman, Acute Myocardial Infarction . 2019, StatPearls Publishing.

3. Carville, S.F., R. Henderson, and H. Gray, The acute management of ST-segment-elevation myocardial infarction. Clinical medicine (London, England), 2015. 15 (4): p. 362-367. 
4. Shi, W.Y. and J.A. Smith, Role of Coronary Artery Bypass Surgery in Acute Myocardial Infarction . 2018, Springer.

5. Raghavan, R., B.S. Benzaquen, and L. Rudski, Timing of bypass surgery in stable patients after acute myocardial infarction. The Canadian journal of cardiology, 2007. 23 (12): p. 976-982.

6. Werner, N., G. Nickenig, and J.-M. Sinning, Complex PCI procedures: challenges for the interventional cardiologist. 2018.107 (2): p. 64-73.

7. Caceres, M. and D.S. Weiman, Optimal timing of coronary artery bypass grafting in acute myocardial infarction. Ann Thorac Surg, 2013.95 (1): p. 365-72.

8. Goldfarb, M., et al., Outcome Reporting in Cardiac Surgery Trials: Systematic Review and Critical Appraisal. Journal of the American Heart Association, 2015. 4 (8): p. e002204-e002204.

9. Spadaccio, C. and U. Benedetto, Coronary artery bypass grafting (CABG) vs. percutaneous coronary intervention (PCI) in the treatment of multivessel coronary disease: quo vadis? - a review of the evidences on coronary artery disease. Annals of cardiothoracic surgery, 2018.7 (4): p. 506-515.

10. Bundhun, P.K., C.M. Yanamala, and F. Huang, Percutaneous Coronary Intervention, Coronary Artery Bypass Surgery and the SYNTAX score: A systematic review and meta-analysis. Scientific reports, 2017.7 : p. $43801-43801$.

11. Baig, K., et al., Does coronary artery bypass grafting improve quality of life in elderly patients? Interactive cardiovascular and thoracic surgery, 2013. 17 (3): p. 542-553.

12. Stone, G.W., et al., Five-Year Outcomes after PCI or CABG for Left Main Coronary Disease. N Engl J Med, 2019. 381 (19): p. 1820-1830.

13. Chang, M., et al., Comparison of Outcome of Coronary Artery Bypass Grafting Versus Drug-Eluting Stent Implantation for Non-ST-Elevation Acute Coronary Syndrome. 2017. 120 (3): p. 380-386.

14. Ngaage, D.L., F. Sogliani, and A. Tang, Early and late prognostic implications of coronary artery bypass timing after myocardial infarction. European Journal of Cardio-Thoracic Surgery, 2012. 43 (3): p. 549-554.

15. Hachet, O., et al., Frequency and predictors of stroke after acute myocardial infarction: specific aspects of in-hospital and postdischarge events. Stroke, 2014. 45 (12): p. 3514-20.

16. Tamer Owais, A.F., Mohamed Adel, Martin Breuer, Jurgen Fuchs, Bernward Lauer, Thomas Kuntze, Timing and Outcome of Coronary Artery Bypass Grafting in STEMI: On-Pump and off-Pump Dilemma. Journal of Cardiology \& Current Research, 2017.

17. Violette, P.D., et al., A cost analysis of nonelective coronary artery bypass graft surgery. J Card Surg, 2006.21 (6): p. 621-7.

18. Sun, L.Y., et al., Long-term Outcomes in Patients With Severely Reduced Left Ventricular Ejection Fraction Undergoing Percutaneous Coronary Intervention vs Coronary Artery Bypass Grafting.2020. 5 (6): p. 631-641.

19. Mølstad, P., R. Moer, and O. Rødevand, Long-term survival after coronary bypass surgery and percutaneous coronary intervention. Open Heart, 2016. 3 (2): p. e000489.

20. Nichols, E.L., et al., Optimal Timing From Myocardial Infarction to Coronary Artery Bypass Grafting on Hospital Mortality.Ann Thorac Surg, 2017. 103 (1): p. 162-171.

21. Gu, Y.L., et al., Role of coronary artery bypass grafting during the acute and subacute phase of STelevation myocardial infarction. Netherlands heart journal : monthly journal of the Netherlands Society of Cardiology and the Netherlands Heart Foundation, 2010. 18 (7-8): p. 348-354. 
22. Grothusen, C., et al., Outcome of Stable Patients With Acute Myocardial Infarction and Coronary Artery Bypass Surgery Within 48 Hours: A Single-Center, Retrospective Experience. Journal of the American Heart Association, 2017. 6 (10): p. e005498.

23. Davierwala Piroze, M., et al., Does Timing of Coronary Artery Bypass Surgery Affect Early and LongTerm Outcomes in Patients With Non-ST-Segment-Elevation Myocardial Infarction? Circulation, 2015.132 (8): p. 731-740.

24. Parikh, S.V., et al., Timing of in-hospital coronary artery bypass graft surgery for non-ST-segment elevation myocardial infarction patients results from the National Cardiovascular Data Registry ACTION Registry-GWTG (Acute Coronary Treatment and Intervention Outcomes Network Registry-Get With The Guidelines) , in JACC Cardiovasc Interv . 2010, 2010 American College of Cardiology Foundation. Published by Elsevier Inc: United States. p. 419-27.

25. Ha, L.D., et al., Early versus delayed coronary artery bypass graft surgery for patients with non-ST elevation myocardial infarction.Coron Artery Dis, 2017. 28 (8): p. 670-674.

26. Assmann, A., et al., Appropriate timing of coronary artery bypass grafting after acute myocardial infarction. Thorac Cardiovasc Surg, 2012. 60 (7): p. 446-51.

27. Wu, C., et al., Risk Score for Predicting Long-Term Mortality After Coronary Artery Bypass Graft Surgery. Circulation, 2012.125 (20): p. 2423-2430.

28. Farkouh, M.E., et al., Strategies for Multivessel Revascularization in Patients with Diabetes. New England Journal of Medicine, 2012. 367 (25): p. 2375-2384.

29. Kapur, A., et al., The Coronary Artery Revascularisation in Diabetes (CARDia) trial: background, aims, and design. Am Heart J, 2005. 149 (1): p. 13-9.

30. Group, B.D.S., A Randomized Trial of Therapies for Type 2 Diabetes and Coronary Artery Disease. New England Journal of Medicine, 2009. 360 (24): p. 2503-2515.

31. Abdallah, M.S., et al., Quality of life after PCI vs CABG among patients with diabetes and multivessel coronary artery disease: a randomized clinical trial. Jama, 2013. 310 (15): p. 1581-90.

32. NICE, Unstable Angina and NSTEMI: the early management of unstable angina and non-ST-segmentelevation myocardial infarction. Clinical guideline 94 . 2013. p. 31, 169.

33. Cardiology, E.S.o., Guidelines on myocardial revascularization . 2018.

34. NICE, Myocardial infarction with ST-segment elevation: the acute management of myocardial infarction withST-segment elevation. Clinical guideline 167 . 2013.

35. Hillis, L.D., et al., 2011 ACCF/AHA Guideline for Coronary Artery Bypass Graft Surgery. A report of the American College of Cardiology Foundation/American Heart Association Task Force on Practice Guidelines. Developed in collaboration with the American Association for Thoracic Surgery, Society of Cardiovascular Anesthesiologists, and Society of Thoracic Surgeons. J Am Coll Cardiol, 2011. 58 (24): p. e123-210.

36. Hu, D., et al., Acute Coronary Syndrome Risk Prediction Based on GRACE Risk Score. Stud Health Technol Inform, 2017. 245 : p. 398-402.

37. society, B.c., Myocardial Ischaemia National Audit Project (MINAP) . 2019.

38. Gale, C.P., et al., Resolving inequalities in care? Reduced mortality in the elderly after acute coronary syndromes. The Myocardial Ischaemia National Audit Project 2003-2010. Eur Heart J, 2012.33 (5): p. 630-9.

39. Toleva, O., et al., Practice patterns and clinical outcomes among non-ST-segment elevation acute coronary syndrome (NSTE-ACS) patients presenting to primary and tertiary hospitals: insights from the EARLY glycoprotein IIb/IIIa inhibition in NSTE-ACS (EARLY-ACS) trial.Catheter Cardiovasc Interv, 2014. 84 (6): p. 934-42. 
40. Hall, M., et al., Association of Clinical Factors and Therapeutic Strategies With Improvements in Survival Following Non-ST-Elevation Myocardial Infarction, 2003-2013. JAMA, 2016.316 (10): p. 1073-82.

41. Lee, D.C., et al., Appropriate timing of surgical intervention after transmural acute myocardial infarction. J Thorac Cardiovasc Surg, 2003. 125 (1): p. 115-9; discussion 119-20.

42. Weiss, E.S., et al., Optimal timing of coronary artery bypass after acute myocardial infarction: a review of California discharge data. J Thorac Cardiovasc Surg, 2008. 135 (3): p. 503-11, 511.e1-3.

43. Mehta, S.R., et al., Early versus delayed invasive intervention in acute coronary syndromes. N Engl J Med, 2009.360 (21): p. 2165-75.

44. Milasinovic, D., et al., Three-Year Impact of Immediate Invasive Strategy in Patients With Non-STSegment Elevation Myocardial Infarction (from the RIDDLE-NSTEMI Study). Am J Cardiol, 2018.122 (1): p. 54-60.

45. Montalescot, G., et al., Effect of prasugrel pre-treatment strategy in patients undergoing percutaneous coronary intervention for NSTEMI: the ACCOAST-PCI study. J Am Coll Cardiol, 2014.64 (24): p. 25632571 .

46. Farkouh, M.E., et al., Impact of Chronic Kidney Disease on Outcomes of Myocardial Revascularization in Patients With Diabetes. J Am Coll Cardiol, 2019. 73 (4): p. 400-411.

47. Najafi, M., M. Sheikhvatan, and S.H. Mortazavi, Do preoperative pulmonary function indices predict morbidity after coronary artery bypass surgery? Annals of cardiac anaesthesia, 2015.18 (3): p. 293-298.

48. Kosmidou, I., et al., C-reactive protein and prognosis after percutaneous coronary intervention and bypass graft surgery for left main coronary artery disease: Analysis from the EXCEL trial. Am Heart J, 2019. 210 : p. $49-57$.

49. Thielmann, M., et al., Predictors and outcomes of coronary artery bypass grafting in ST elevation myocardial infarction. Ann Thorac Surg, 2007. 84 (1): p. 17-24.

50. Ng, V.G., et al., The prognostic importance of left ventricular function in patients with ST-segment elevation myocardial infarction: the HORIZONS-AMI trial. European heart journal. Acute cardiovascular care, 2014. 3 (1): p. 67-77.

51. Yang, T., et al., Myocardial scar identified by magnetic resonance imaging can predict left ventricular functional improvement after coronary artery bypass grafting. PloS one, 2013. 8 (12): p. e81991-e81991.

52. Sheriff, M.J., et al., Cardiac Magnetic Resonance Imaging Predictors of Short-Term Outcomes after High Risk Coronary Surgery.2016. 25 (6): p. 613-619.

53. Nucifora, G. and J.B. Selvanayagam, Cardiac Magnetic Resonance Imaging Before Coronary Artery Bypass Graft Surgery: Is It Ready for Risk Stratification? 2016. 25 (6): p. 535-537.

54. Hausenloy, D.J., et al., The effect of cyclosporin-A on peri-operative myocardial injury in adult patients undergoing coronary artery bypass graft surgery: a randomised controlled clinical trial.Heart, 2014. 100 (7): p. 544. 
Table 1: comparison of advantages and disadvantages of CABG and PCI (9)

\begin{tabular}{|c|c|c|c|}
\hline \multicolumn{2}{|c|}{ CABG } & \multicolumn{2}{|c|}{$\mathbf{P C l}$} \\
\hline Advantages & Disadvantages & Advantages & Disadvantages \\
\hline $\begin{array}{l}\text { Prolong life in stable } \\
\text { coronary disease }\end{array}$ & More invasive & Less invasive & $\begin{array}{l}\text { Cath lab is not always } \\
\text { accessible }\end{array}$ \\
\hline $\begin{array}{l}\text { CABG bypasses } \\
\text { existing lesion and } \\
\text { future threatening } \\
\text { lesions }\end{array}$ & $\begin{array}{l}\text { Scar marks from open } \\
\text { surgery }\end{array}$ & No scar marks & $\begin{array}{l}\text { Stent does not } \\
\text { address future lesions }\end{array}$ \\
\hline $\begin{array}{l}\text { Can treat multiple } \\
\text { lesions }\end{array}$ & Longer hospital stays & Shorter hospital stays & $\begin{array}{l}\text { Potential incomplete } \\
\text { revascularisation and } \\
\text { restenosis }\end{array}$ \\
\hline $\begin{array}{l}\text { Decrease recurrence } \\
\text { of angina }\end{array}$ & $\begin{array}{l}\text { Later work } \\
\text { resumption }\end{array}$ & Early work resumption & $\begin{array}{l}\text { Increase recurrence of } \\
\text { angina }\end{array}$ \\
\hline $\begin{array}{l}\text { Achieve complete } \\
\text { revascularisation }\end{array}$ & $\begin{array}{l}\text { High risk of } \\
\text { mortality/morbidity in } \\
\text { repeated CABG }\end{array}$ & Lower rate of stroke & $\begin{array}{l}\text { High risk of repeated } \\
\text { revascularisation }\end{array}$ \\
\hline
\end{tabular}


Table 2: Predictive factors of CABG outcome $(27,48,49,54)$

Outcome

Predictive factors of $C A B G$

\begin{tabular}{|c|c|}
\hline Positive & $\begin{array}{l}\text { Preoperative measures: } \\
\text { - Dual antiplatelet therapy until surgery } \\
\text { - Cyclosporin-A administered prior to } \\
\text { surgery }\end{array}$ \\
\hline & $\begin{array}{l}\text { Pulmonary function: } \\
\begin{array}{l}\text { - Predicted inspiratory vital capacity of } \\
\geq 75 \% \\
\text { - } \\
\text { Predicted maximal expiratory pressure } \\
\text { of } \geq 75 \%\end{array}\end{array}$ \\
\hline Negative & 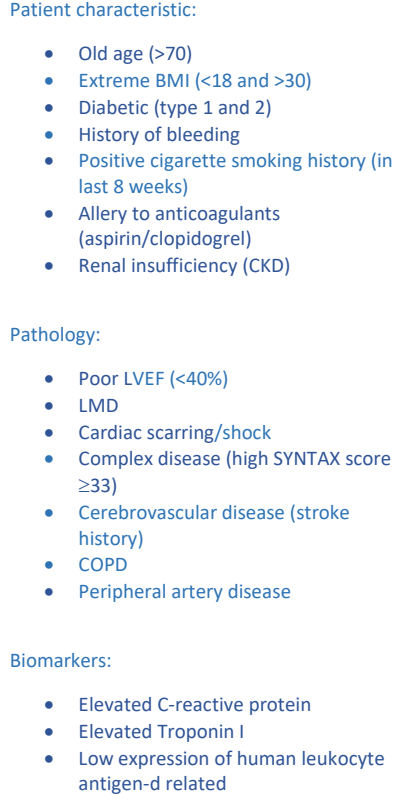 \\
\hline
\end{tabular}

